\title{
Massively Parallel and Linear-Scaling Algorithm for Second-Order Møller-Plesset Perturbation Theory Applied to the Study of Supramolecular Wires
}

\author{
Thomas Kjærgaard ${ }^{\mathrm{a}, *}$, Pablo Baudin ${ }^{\mathrm{a}}$, Dmytro Bykov ${ }^{\mathrm{a}}$, Janus Juul Eriksen ${ }^{\mathrm{a}}$, Patrick Ettenhuber ${ }^{\mathrm{a}}$, Kasper Kristensen ${ }^{\mathrm{a}}$, Jeff \\ Larkin $^{\mathrm{d}}$, Dmitry Liakh ${ }^{\mathrm{b}}$, Filip Pawłowski ${ }^{\mathrm{a}}$, Aaron Vose ${ }^{\mathrm{c}}$, Yang Min Wang ${ }^{\mathrm{a}}$, Poul Jørgensen ${ }^{\mathrm{a}}$ \\ ${ }^{a}$ qLEAP Center for Theoretical Chemistry, Department of Chemistry, Aarhus University, Langelandsgade 140, DK-8000 Aarhus C, Denmark \\ ${ }^{b}$ Scientific Computing Group, National Center for Computational Sciences, Oak Ridge National Laboratory, Oak Ridge, TN, 37831, USA \\ ${ }^{c}$ Cray Inc. \\ ${ }^{d}$ NVIDIA Inc.
}

\begin{abstract}
We present a scalable cross-platform hybrid MPI / OpenMP / OpenACC implementation of the Divide-Expand-Consolidate (DEC) formalism with portable performance on heterogeneous HPC architectures. The Divide-Expand-Consolidate formalism is designed to reduce the steep computational scaling of conventional many-body methods employed in electronic structure theory to linear scaling, while providing a simple mechanism for controlling the error introduced by this approximation. Our massively parallel implementation of this general scheme has three levels of parallelism, being a hybrid of the loosely coupled task-based parallelization approach and the conventional MPI+X programming model, where X is either OpenMP or OpenACC. We demonstrate strong and weak scalability of this implementation on heterogeneous HPC systems, namely on the GPU-based Cray XK7 Titan supercomputer at the Oak Ridge National Laboratory. Using the "resolution of the identity second-order Møller-Plesset perturbation theory" (RI-MP2) as the physical model for simulating correlated electron motion, the linear-scaling DEC implementation is applied to 1-aza-adamantane-trione (AAT) supramolecular wires containing up to 40 monomers (2440 atoms, 6800 correlated electrons, 24440 basis functions and 91280 auxiliary functions). This represents the largest molecular system treated at the MP2 level of theory, demonstrating an efficient removal of the scaling wall pertinent to conventional quantum many-body methods.
\end{abstract}

\section{Introduction}

In recent decades, quantum-mechanical electronic structure calculations have become an integral part of many disciplines of molecular sciences, e.g., chemistry, physics, material science, atomically precise manufacturing, molecular biology, and pharmacology. Electronic structure calculations have thus become an invaluable tool for the interpretation and rationalization of experimental results. Additionally, electronic structure calculations have also been proven important due to their predictive power, for example, in the design of new molecular structures and materials with desired properties. The reliability of such predictions increases with an increasing accuracy of the electronic structure calculations. Consequently, the development of high-accuracy electronic structure models has therefore become a task of high priority.

In electronic structure calculations, the electronic Schrödinger equation is solved approximately because of its unfavorable computational scaling. For smaller molecular systems, the coupled-cluster (CC) model is the state-of-the-art wave-function method, and a hierarchy of approximate CC models-MP2[1] (second-order Møller-Plesset perturbation theory), CCSD[2] (coupled cluster with singles and doubles), and $\operatorname{CCSD}(\mathrm{T})[3]$ (coupled cluster with singles, doubles, and perturbative triples) has been established, where the exact solution to the Schrödinger equation is approached in a systematic manner, and the accuracy of the calculations hence systematically controlled. Using the CC hierarchy of approximate models, energy levels, structures, and many molecular properties of small molecules can be computed to an accuracy challenging experimental results [4].

The standard formulation of the CC model hierarchy exhibits a steep polynomial computational scaling with the system size (number of simulated particles), which for many years has hindered the CC hierarchy from being applied to studying large mblecular systems. For this reason, electronic structure calculations of large molecular systems have for many years

exists, however no clue is given to a systematic way for determining this functional. DFT is therefore a semi-empirical theory where a physical intuition and performance statistics have been guiding the development of new functionals, all with "improved performance". Although successful and computationally efficient, the DFT approach may always be questioned for its reliability. In the long run it is "a must" for molecular sciences to have the capability of obtaining rigorous highaccuracy solutions to the electronic Schrödinger equation. At the same time, the corresponding high-accuracy many-body

\footnotetext{
* Corresponding author

Email address: tkjaergaard@chem.au.dk (Thomas Kjærgaard)
} 
methods should still stay computationally affordable. Ideally a linear scaling of the computational cost with the system size is desired. Below we demonsrate how this goal can be achieved using the Divide-Expand-Consolidate formalism [5, 6, 7]. An important point is that the linear-scaling regime is activated only after some critical system size, thus requiring large-scale computational resources in order to get there. Consequently, a scalable parallel implementation of the DEC formalism is absolutely necessary in order to efficiently simulate large molecular systems. Taking into account the probable architecture of future large-scale HPC platforms, which is likely to be heterogeneous, the goal of this paper is to describe our massively parallel, accelerator-enabled implementation of the DEC formalism and to demonstrate its scalability on an existing largescale heterogeneous HPC system, namely the GPU-based Cray XK7 Titan supercomputer located at the Oak Ridge National Laboratory.

The specific physical model we focus on in this paper is the lowest member of the CC hierarchy, the MP2 model. The computational cost of a conventional MP2 formulation scales as $k N^{5}$, where $N$ is the number of one-electron basis functions in the calculation and $k$ is a prefactor. The complexity of a conventional MP2 formulation may be reduced using two different strategies: (i) reducing the prefactor $k$, and (ii) reducing the $N^{5}$ scaling to a lower power, ideally to a linear dependence on $N$. Both of these strategies require that approximations are introduced in the conventional MP2 model.

The prefactor $k$ may be reduced using the RI approximation [8, 9, 10, 11, 12, which is a standard approximation used for reduction of the complexity of the conventional MP2 formulation that has led to the RI-MP2 method [13, 14, 15, 16, 17, $18,19,20,21,22$. In the RI approximation, a resolution of the identity is introduced to replace the evaluation of four-center two-electron repulsion integrals (ERIs) by evaluation of two- and three-center integrals. The error of the RI approximation is fully controllable via the size and design of the auxiliary basis in which the resolution of the identity is expanded. In the limit of a complete (infinite) auxiliary basis, the conventional MP2 energy is thus recovered. As an alternative to the RI approximation, a Cholesky decomposition [23, 24, 25] of the ERIs may be performed. Massively parallel implementations have been reported for the RI-MP2 method [14, 16. However, these implementations do not provide a linear scaling of the computational cost and the corresponding calculations will inevitably hit the scaling wall when the size of the system is increased. Thus, a massively parallel implementation of the brute-force RI-MP2 approach only moderately extends its application range.

In practice it turns out that the $N^{5}$ scaling of the conventional MP2 algorithm may be reduced by exploiting the local nature of the electron correlation. The corresponding local correlation methods [26, 27, are formulated either in the atomic orbital (AO) basis 28, 29, 30, or in a basis of localized molecular orbitals (MOs) [31, 32, 33, 34, 35, 36, 37, 38, 39, 40, 41, 42, 43, 44. Our recently introduced Divide-Expand-Consolidate (DEC) strategy [5, 6, 7] is a local MO-based correlation approach, in which a calculation of a large molecule is partitioned into a large number of smaller atomic fragment and pair fragment calculations. It should be emphasized that a DEC calculation does not involve any physical fragmentation of the molecule, but merely a partitioning of the local orbital space. In principle, the number of pair fragments scales quadratically with the system size; however, due to the $R^{-6}$ decay of the dispersion energies with the inter-fragment distance, $R$, distant pairs may be neglected without compromising the accuracy of the calculation [5, 6, ultimately leading to a linear-scaling algorithm. As the calculations of individual fragments are independent of one another, DEC is also a massively parallel algorithm [45] by construction. The fragments and their sizes are dynamically determined in a black-box manner, such that the error due to partitioning of a molecule into fragments is fully controlled via a single parameter, the fragment optimization threshold (FOT). The precision of the DEC calculation may therefore be systematically increased by decreasing the FOT. In the limiting case of the FOT approaching zero, the conventional MP2 result is recovered. Combining our DEC strategy with the RI-MP2 model defines the DEC-RI-MP2 algorithm [46.

Other local MO-based MP2 correlation methods include the local coupled-cluster method of Werner and co-workers 32,47 , 48 and the domain-based local pair-natural-orbital coupled-cluster (DLPNO-CC) method of Neese and co-workers 36, 44. In these approaches a full molecular CC equation is solved where the excitation space for each pair of occupied orbitals is assigned a restricted local virtual orbital space, which is defined in terms of fixed (i.e., not dynamical) thresholds. The default values for these fixed thresholds have been determined empirically, and the precision of the correlation energy compared to a conventional calculation is therefore in general unknown. In addition to DEC, there exist other local correlation methods relying on a partitioning of the orbital space rather than on a physical fragmentation of the molecule, e.g., the cluster-in-molecule method [49, 43] and the incremental scheme [50, 35. However, only DEC uses a dynamical adaptation of orbital fragment spaces to a predefined energy error. On the contrary, the Divide-and-Conquer MP2 [51, 52] and the Fragment Molecular Orbital MP2 (FMO-MP2) [53, 54] are two examples of a myriad of molecular fragmentation approaches [55, 56, 57, 58, 59, 60. In these methods, a physical fragmentation of a molecular system into fragments is performed based on a "chemical intuition," making it impossible to estimate the error introduced compared to a conventional MP2 calculation 22 .

The other group of local correlation approaches, for example the AO-based MP2 formulations, usually employ a Laplace transformation 61, 62, where the orbital energy denominator is eliminated from the conventional MO-based MP2 energy expression to obtain a formulation in terms of AO integrals [28, 63, 64, 65. Since AOs are local by construction, efficient integral screening may be performed, where an $R^{-4}$ decay may be exploited when ERIs are evaluated. However, as the integral decay $\left(R^{-4}\right)$ is slower than the dispersion energy decay $\left(R^{-6}\right)$, the locality of electronic dispersion effects is not fully exploited in the Laplace AO-based MP2 methods.

In this article we report a massively parallel linear-scaling DEC-RI-MP2 implementation enhanced with the accelerator support, where our DEC strategy is used to reduce the steep $N^{5}$ scaling of the MP2 method to linear scaling $(N)$ and the 
RI approximation is used for reducing the prefactor $k$ for individual fragment calculations. The DEC-RI-MP2 calculations are carried out for the stacked 1-aza-adamantane-trione supramolecular wires containing up to 40 monomers. The largest calculation comprises 2440 atoms, 6800 correlated electrons, 24440 basis functions and 91280 auxiliary functions. It ran on 14952 out of 18688 nodes of the Titan supercomputer, a GPU-based Cray XK7 HPC platform which is currently positioned \# 3 in the TOP500 list. The presented calculation is 2.5 times larger than the largest conventional RI-MP2 calculation [16. We also demonstrate the weak and strong parallel scalability of our DEC algorithm.

\section{Theory}

In the DEC-RI-MP2 scheme, localized occupied and unoccupied Hartree-Fock (HF) orbitals 66, 67, 68, 69, 70, 71, 72, 73, 74, 75, 76, 77, 78, 79, 80, 81, 82 are used to efficiently describe the short-ranged electron correlation effects, partitioning the calculation into many small and independent fragment calculationsa and ultimately obtaining a linear-scaling algorithm (for a recent review on the characterization and generation of local occupied and virtual HF orbitals see Ref 83).

In this section, we summarize the DEC-RI-MP2 algorithm and refer the reader to Ref. 46 for additional details. The first step in the DEC scheme is to assign each localized $\mathrm{MO}$ to an atomic site $(P, Q, \ldots)$. Each site $P$ thus gets assigned a set of occupied, $\underline{P}$, and unoccupied, $\bar{P}$, localized orbitals.

The RI-MP2 energy is the sum of the HF contribution, $E_{\mathrm{HF}}$, which represents the mean-field electronic interactions, and the correlation contribution, $E_{\text {corr }}$, which represents the dynamical correlation among the electrons. In conventional implementations, the determination of the correlation energy is the time-dominating part, and we therefore focus of on $E_{\text {corr }}$ here and refer the reader to Refs. 84, 85, 86, 87, 88, 89, 90, 91, 92, 93, 94 for linear-scaling HF algorithms. In a DEC-RI-MP2 calculation, the correlation energy is partitioned into independent fragment calculations according to

$$
E_{\text {corr }}=\sum_{P} E_{P}+\sum_{P>Q} \Delta E_{P Q},
$$

where the atomic fragment energy $E_{P}$ and the pair fragment energy $\Delta E_{P Q}$ are given by,

$$
\begin{aligned}
E_{P} & =\sum_{i j \in \underline{P}} \sum_{a b \in[\bar{P}]} t_{a i b j}\left(2 g_{a i b j}-g_{a j b i}\right), \\
\Delta E_{P Q} & =\sum_{\substack{i \in \underline{P} \\
j \in \underline{Q}}} \sum_{a b \in[\bar{P}] \cup[\bar{Q}]} t_{a i b j}\left(2 g_{a i b j}-g_{a j b i}\right) \\
& +\sum_{\substack{i \in \underline{Q} \\
j \in \underline{P}}} \sum_{a b \in[\bar{P}] \cup[\bar{Q}]} t_{a i b j}\left(2 g_{a i b j}-g_{a j b i}\right) .
\end{aligned}
$$

Here, $g_{a i b j}$ and $t_{a i b j}$ are ERIs and RI-MP2 wave function amplitudes, respectively, expressed in the local orbital basis. The amplitudes and ERIs for the atomic fragment $P$ are determined using a set of occupied MOs $[\underline{P}]$, unoccupied MOs $[\bar{P}]$, standard AOs $\{P\}_{\mathrm{AO}}$, and auxillary AOs $\{P\}_{\text {aux }}$ spatially close to $P$, see Fig. 1 for a schematic illustration and Table 1 for an overview of the notation. The MOs are expanded in the $\{P\}_{\mathrm{AO}}$ space, and the $\{P\}_{\text {aux }}$ space is used for the RI approximation of the ERIs.

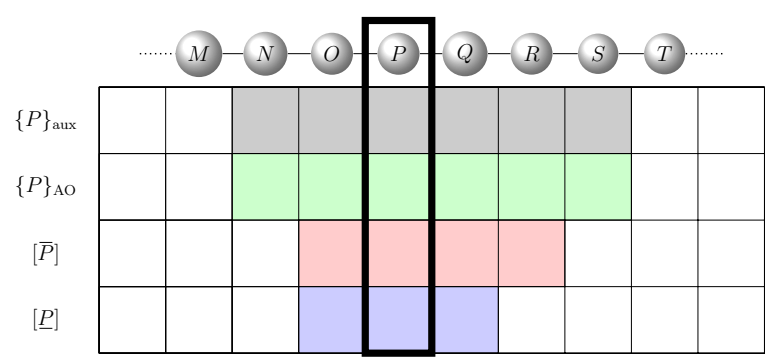

Figure 1. Illustration of an atomic fragment $P$. The string of spheres is a schematic representation of a molecule with each sphere representing an atom. The local orbital spaces of occupied MOs $[\underline{P}]$ (blue), unoccupied MOs $[\bar{P}]$ (red), standard AOs $\{P\}_{\text {AO }}$ (green), and auxillary AOs $\{P\}_{\text {aux }}$ (grey) are indicated. The blue and red square encapsulated by the bold black box represents the spaces $\underline{P}$ and $P$. 
Table 1. Notation for indices and dimensions of different local orbital spaces for an atomic fragment $P$.

\begin{tabular}{lll}
\hline \hline Explanation & Notation & Dim. \\
\hline Occupied MO, assigned to $P$ & $i, j \in \underline{P}$ & $\bar{O}$ \\
Unoccupied MO, assigned to $P$ & $a, b \in \bar{P}$ & $\bar{V}$ \\
Occupied MO local space & $i, j \in[\underline{P}]$ & $O$ \\
Unoccupied MO local space & $a, b \in[\bar{P}]$ & $V$ \\
Occupied pseudo-canonical & $I, J \in[\underline{P}]$ & $O$ \\
Unoccupied pseudo-canonical & $A, B \in[\bar{P}]$ & $V$ \\
Standard AO local space & $\mu \in\{P\}$ & $N$ \\
Auxillary AO local space & $\alpha \in\{P\}_{\text {aux }}$ & $N_{\text {aux }}$ \\
\hline \hline
\end{tabular}

If one removes the restrictions in the summations over unoccupied orbitals in Eqs. (2) and (3), the standard RI-MP2 correlation energy is obtained, preventing any reduction in computational cost. However, using the locality of the orbitals (and hence of the ERIs and amplitudes), the orbital spaces $[\bar{P}]$ can be restricted to a small subset of the complete orbital space without affecting significantly the precision of the calculation while resulting in large computational savings. In practice, these local spaces are determined in a black box manner using a single parameter, the FOT [6]. The resulting fragment energy $E_{P}$ is thus controlled by the FOT and ensured to reach a user-defined accuracy. The value of the FOT therefore determines the overall accuracy and the computational cost of a DEC-RI-MP2 calculation. While the number of atomic fragments scales linearly with system size, the number of pair fragments scales quadratically with the system size; however, due to the rapid decay of dispersion energies(inverse pair distance to the sixth power [95]), distant pairs may be neglected without compromising the precision of the calculation [6], ultimately leading to a linear-scaling algorithm.

In summary, a DEC-RI-MP2 energy calculation proceeds as follows:

1. First, the HF information is read (Fock matrix and localized MOs).

2. The orbital spaces $[\bar{P}]$ are optimized to provide atomic fragment energies, $E_{P}$, to the FOT precision.

3. A reduced set of pair fragments is selected based on estimations of their energy contribution (pair estimate calculations).

4. The pair fragment energies are computed for the selected pairs using Eq. (3) (this is the most expensive step).

5. Finally, the total correlation energy is calculated using Eq. (1).

There are three parallelization levels in the DEC scheme 45: (1) coarse-grained parallelization, where independent fragment calculations (tasks) are distributed among groups of MPI processes, (2) medium-grained parallelization, where each fragment calculation is parallelized across a number of nodes constituting an MPI process group, and (3) fine-grained parallelization (OpenMP/OpenACC parallelization across the CPU/GPU cores on each node).

At the coarse-grained parallelization level, the independent tasks (individual and pair-fragment calculations) are sorted according to their size. The largest tasks are scheduled first, being distributed among MPI process groups. As the tasks become smaller in size, the original groups of MPI processes may split into smaller groups such that the size of each MPI process group is appropriate for the size of the tasks it is executing. The task based workflow together with the dynamic execution unit granularity adjustment ensures load balancing at the coarse-grained parallelization level and efficiency at the medium-grained parallelization level.

Regarding the medium-grained parallelism, each (atomic or pair) fragment can be seen as a standard RI-MP2 calculation in a reduced orbital space and can be efficiently parallelized over a number of nodes. The evaluation of Eqs. (2) and (3) requires the determination of the local amplitudes $t_{a i b j}$. In order to obtain a non-iterative algorithm these amplitudes are first determined in a so-called pseudo-canonical basis $t_{A I B J}$, which is generated by diagonalizing the local Fock matrices $\left(F_{i j}\right.$ with $i j \in[\underline{P}]$ and $F_{a b}$ with $\left.a b \in[\bar{P}]\right)$. Denoting the occupied/unoccupied diagonal elements of the Fock matrix in the pseudo-canonical basis as $\left(\epsilon_{I}, \epsilon_{J}\right) /\left(\epsilon_{A}, \epsilon_{B}\right), t_{A I B J}$ is evaluated in the following step, which is the most expensive and scales as $N_{\text {aux }} V^{2} O^{2}$ (see Table 1),

$$
t_{A I B J}=\frac{g_{A I B J}}{\epsilon_{I}+\epsilon_{J}-\epsilon_{A}-\epsilon_{B}}=\frac{\sum_{\alpha} C_{A I}^{\alpha} C_{B J}^{\alpha}}{\epsilon_{I}+\epsilon_{J}-\epsilon_{A}-\epsilon_{B}},
$$

where the RI approximation has been used to express the ERIs in terms of fitting coefficients $C_{A I}^{\alpha}$. The pseudo-canonical amplitudes and ERIs are subsequently transformed back to the local basis $\left(t_{A I B J} \rightarrow t_{a i b j}\right)$ for the evaluation of the fragment energy using Eqs. (2) or (3). Most of the computations required to obtain the fragment energies (e.g., Eq. (4P) can be performed by threaded/accelerated optimized libraries (BLAS) or compiler directive parallelized kernels (OpenMP on the CPU or OpenACC on the GPU). This corresponds to the fine-grained level of parallelization. The construction of the ERIs and the amplitudes, as well as the subsequent transformation from the pseudo-canonical to the local basis are thus performed on the GPU, while the fitting coefficients are currently constructed using a CPU only code.

In Fig. 2, the coarse-grained parallelism of DEC is exemplified for a calculation containing 12 fragments and using 9 nodes. Node 0 is the global master node (blue), and the remaining nodes are divided into slots each containing 4 nodes, where one of the nodes is chosen as the local master (red) and the remaining nodes are referred to as local slaves (grey). A 


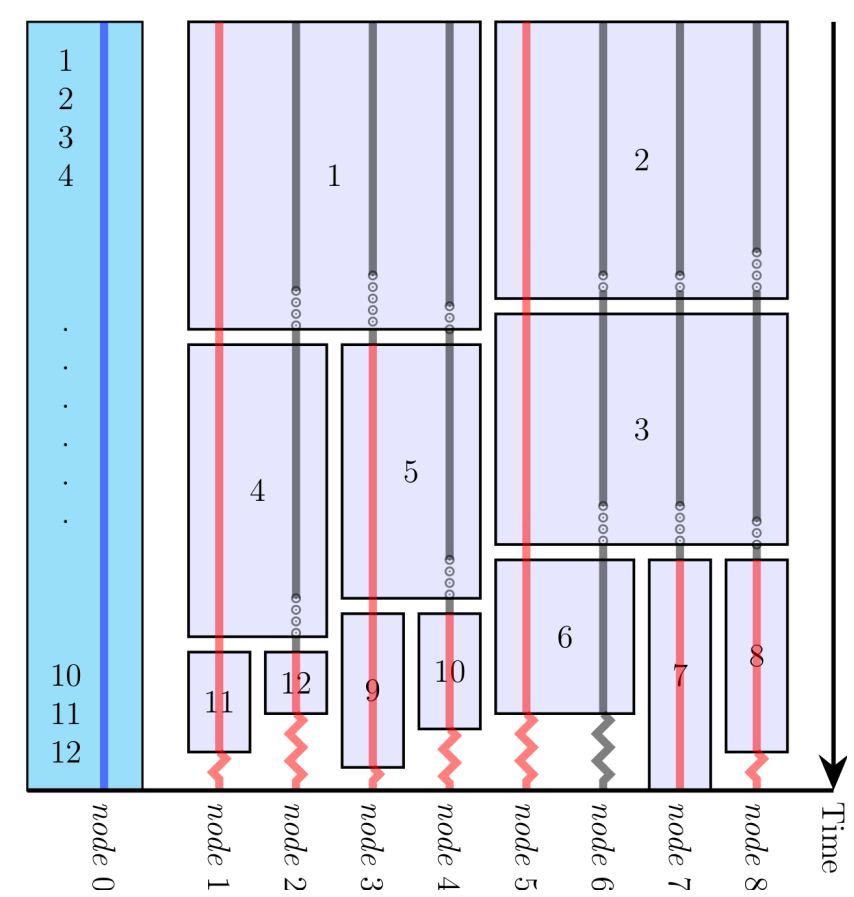

Figure 2. Coarse-grained parallelization of the DEC scheme exemplified by a calculation with 12 fragments using 9 nodes. The fragment jobs are ordered according to size in descending order on the global master (blue). The local masters (red) receive job instructions, carry out job in collaboration with the local slaves (grey) in the slot, and send the fragment energy to the global master. The slots divide dynamically to ensure good medium-grained parallelization, see text.

Although the ordering of fragments according to size and the splitting of slots according to Eq. (5) is an attempt to minimize the idle time, some loss is to be expected, and we distinguish between local (dotted lines) and global (wiggly lines) loss of efficiency in Fig. 2. The local loss is associated with the medium-grained parallelization and is present due to non-ideal load balancing among the local working nodes (local master and local slaves), while the global loss occurs when all jobs have been distributed by the global master and some of the nodes wait for the remaining jobs to finish.

\section{Molecular systems used to measure performace}

As an example application for the DEC-RI-MP2 method, we consider a new class of molecules, the 1-aza-adamantanetriones (AATs) [96, 97]. AATs spontaneously self-assemble into ordered nano-structures, and their electronic and physical properties can be experimentally tuned. Therefore, AATs are promising model materials, and in their supramolecular wire form they have potential applications in the fields of molecular electronics and optoelectronics. The intrinsic forces involved in the nanoscale self-assembly of these supramolecular structures are dominated by dispersion forces and $\pi-\pi$ stacking interactions, that can only be accurately described using correlated methods. We will apply the DEC-RI-MP2 model to calculate interaction energies of AATs using the correlation-consistent cc-pVDZ basis set and the auxiliary cc-pVDZ-RI basis set 98. We will 
present scientific results and error analysis of the DEC-RI-MP2 calculations for up to 10 monomers here and in the performance section we will demonstrate the computational efficiency of DEC-RI-MP2 by carrying out calculations for up to 40 stacked AATs, which is far beyond what can be done using a conventional implementation.

The $\mathrm{AAT}_{n}$ structure for a general $n$-mer was obtained by duplicating and translating the optimized $\mathrm{AAT}_{2}$ structure $(n / 2-1)$ times, such that the distance between the two central nitrogen atoms in two adjacent AAT units was the same as 6 in the optimized dimer $(5.12 \AA)$, see Fig. 3. The interaction energy for $A A T_{n}\left(\Delta E_{A A T_{n}}\right)$ was then calculated as the difference 7 in energy between the $A A T_{n}$ system and the two noninteracting $\mathrm{AAT}_{n-1}$ and $A A T_{1}$ systems,

$$
\Delta E_{A A T_{n}}=E_{A A T_{n}}-E_{A A T_{n-1}}-E_{A A T_{1}}
$$

The $E_{A A T_{n-1}}$ and $E_{A A T_{1}}$ monomer calculations were corrected for basis set superposition errors using the counterpoise correction 99]. The resulting interacting energies are given in Table 2 for HF, conventional RI-MP2, and DEC-RI-MP2 where we have used a FOT of $10^{-5} \mathrm{a} . \mathrm{u} .=0.272 \mathrm{meV}$ for the DEC calculations. The DEC errors compared to the conventional RI-MP2 calculations are also presented.

Table 2. Interaction energies $\Delta E_{A A T_{n}}$ for $n=2,4,6,8,10$ calculated using the HF, conventional RI-MP2 (conv), and DEC-RI-MP2 models (using the cc-pVDZ basis). We also give the absolute errors of the DEC interaction energy and the DEC total energy compared to the conventional reference numbers with the recoveries of the correlation contribution $\left(E_{\mathrm{corr}}^{\mathrm{DEC}} / E_{\mathrm{corr}}^{\mathrm{conventional}}\right)$ given in parenthesis. All results are given in $\mathrm{eV}$.

\begin{tabular}{llllll}
\hline \hline$n$ & HF & Conv & DEC & Interaction error & Total error \\
\hline 2 & 0.729 & -0.718 & -0.710 & $0.008(99.43 \%)$ & $0.090(99.97 \%)$ \\
4 & 0.721 & -0.746 & -0.710 & $0.036(97.52 \%)$ & $0.246(99.95 \%)$ \\
6 & 0.716 & -0.748 & -0.724 & $0.023(98.41 \%)$ & $0.382(99.95 \%)$ \\
8 & 0.716 & -0.748 & -0.727 & $0.023(98.43 \%)$ & $0.528(99.95 \%)$ \\
10 & 0.716 & & -0.724 & & \\
\hline \hline
\end{tabular}

The HF model does not describe dispersion forces and it therefore wrongly predicts the interaction energies to be positive, i.e., the AAT units repel each other at the HF level of theory. On the contrary, the conventional RI-MP2 interaction energies are negative (attractive) and thus describe the correct physics due to the RI-MP2 correlation contribution, which represents dispersion effects. An inspection of the DEC errors in Table 2 shows that the DEC error of the total energy $E_{A A T_{n}}$ increases linearly with system size, as expected, while the recovery of the conventional correlation energy is roughly constant at $99.95 \%$. More importantly, the interaction energy error does not change significantly with system size. In fact, for the largest systems, the interaction energy error is more than an order of magnitude smaller than the total error-e.g., for AAT 8 , the total error is $0.528 \mathrm{eV}$, while the error in the interaction energy is only $0.023 \mathrm{eV}$. In short, the DEC-RI-MP2 scheme is capable of accurately reproducing the conventional results.

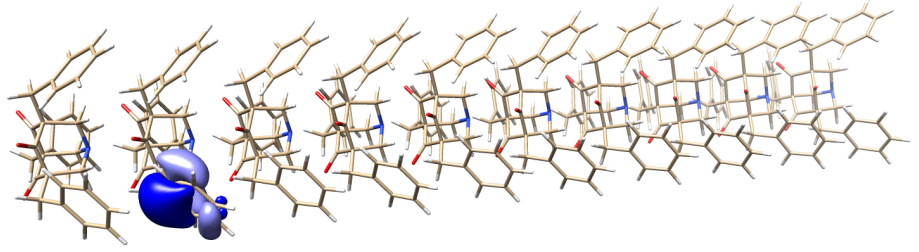

Figure 3. Illustration of $\mathrm{AAT}_{10}$ with one of the localized molecular orbitals shown in blue.

\section{System and environment where performance was measured}

The experimental platform is the Titan supercomputer, a Cray XK7 system deployed by the US Department of Energy at the Oak Ridge National Laboratory, which currently occupies the third position on the Top500 list. The machine comprises 18688 nodes, each consisting of a 16-core AMD Opteron 6274 processor with $32 \mathrm{~GB}$ of main memory and an NVIDIA Tesla K20x GPU accelerator (Kepler architecture) with 6GB of GDDR5 memory. Titan's nodes are connected according to a $3 \mathrm{D}$ torus topology with the Cray Gemini network interfaces. The application-level communication layer is provided by the Cray MPI library implementing most of the MPI 3.0 features, a derivative of MPICH-2 from Argonne National Laboratory. The OpenMP v.3.0 and OpenACC v.2.0 run-times implemented in Cray Compiler Environment v.8.4.2 are used for CPU multi-threading and GPU acceleration, respectively. The Cray OpenACC implementation available on Titan is specifically optimized for Nvidia Kepler GPU architecture. Finally, the Cray LibSci v.13.2.0 scientific library provides optimized versions of linear algebra operations, namely the dgemm and dsyev routines (double precision arithmetic was used throughout the calculations). 
We have measured the number of floating point operations (FLOP\$2 performed on the CPU using the PAPI library [100. The FLOPs performed on the GPU were not directly measured, instead they were estimated using a CPU compiled version of the GPU kernels and analyzed using PAPI. During the performance calculations an estimated GPU FLOP count was will perform the same number of FLOPs. To validate the estimated GPU FLOP count we used $A A T_{4}$ as a test system and

6 found that the CPU PAPI-counted and GPU estimated FLOPs of the hybrid CPU/GPU code corresponded to $99.5 \%$ of the 7 FLOPs measured by PAPI for the CPU-only code.

8 Efficient and lightweight checkpointing was added for the completed fragments, in the sense that the global master 199 simply writes a file with the fragment energy and identity of the completed fragments. This limits I/O but means that 10 each interrupted fragment calculation has to be restarted from scratch. During the performance calculations, Titan suffered 11 from a higher than normal rate of node failures, and checkpointing was crucial for successful completion of the calculations. 12 As the massively parallel architectures have an ever increasing number of nodes and thus risk of node failures, MPI fault 13 tolerance is increasingly important. DEC is set up to exploit future MPI fault tolerance mechanisms, once this is part of the 104 MPI standard, by reassigning the affected fragment to the available nodes. The checkpointing also allows for the fragment 15 optimization and pair estimate calculations to be run using one set of nodes, while a larger set of nodes can be used for the 16 computationally more demanding pair fragment calculations.

All results reported in Section 5 used the same value of the main DEC threshold (FOT=10 ${ }^{-5}$ a.u.) as in Section 3 and the calculated correlation energies are therefore highly accurate (expected 99.95\% recovery of the conventional RI-MP2 correlation energy). The weak scaling analysis in Section 5 was performed for the full application including I/O (step 1-5 in Section 2 while the strong scaling analysis was performed for the time-dominating part only, the pair fragment calculations (step 4 in Section 2). We further emphasize that the construction of the localized HF orbitals is not part of the DEC application.

The DEC code is part of the LSDALTON program 101 and the source code is distributed free of charge as a part of the DALTON2016 suite [102]. The LSDALTON program have been used to construct the localized HF orbitals.

\section{Performance results}

In Table 3 and Fig. 4 we show strong scalability results for $A A T_{10}$ starting with 3738 nodes. Efficiencies from $74.8 \%$ to $94.3 \%$ are observed. The efficiency is quite remarkable considering the complexity of the problem. The $A A T_{10}$ calculation consists of 16023 pair fragments and we used an initial slot size of 32 nodes resulting in (14952/32) 467 slots (see Fig. 2 for an illustrative example of 12 fragments, 9 nodes, and 2 initial slots with a slot size of 4 nodes). Due to the inhomogeneous fragment sizes (ranging from 1106 to 9366 auxiliary fuctions) and the fifth-order scaling with fragment size, the time for the individual fragment calculations ranges from 6 seconds using a single node to 1 hour and 37 minutes using 32 nodes.

Table 3. Strong scalability results on Titan for $A A T_{10}$ using 3738 to 18400 nodes. TTS denotes the Time-To-Solution and the last column denote how many times a calculation was restarted due to hardware failures.

\begin{tabular}{cccc}
\hline \hline \# nodes & TTS(s) & efficiency & restarted \\
\hline 3738 & 39400 & & 1 \\
7476 & 20900 & 94.3 & 0 \\
11214 & 15400 & 85.3 & 0 \\
14952 & 12100 & 81.5 & 1 \\
18400 & 10700 & 74.8 & 2 \\
\hline \hline
\end{tabular}

${ }^{2}$ FLOPs will denote FLOP in plural, while we will use FLOPs/s to denote FLOPs per second. Also, we use 1 EFLOPs $=10^{18}$ FLOPs. 
The efficiency is particular impressive considering that the 14952 node calculation had to be restarted once and the 18400 node calculation twice due to hardware failures and each restart results in an increased global loss (see Fig. 22). Due to the short TTS for the large node jobs, hardware crashes becomes increasingly detrimental for the large node jobs.

Load balancing and job distribution are crucial for efficient strong scaling. For instance, in the case of the 18400 node job, the 1 hour and 37 minutes fragment calculation constitutes $54 \%$ of the total wall time. If a larger number of nodes is used the strong scaling performance will eventually be limited by the time for this fragment. The time for the largest fragments can in this case be reduced by using more than 32 nodes in each slot (see Fig. 5). The larger slot sizes improve the strong scaling by reducing the global loss, but the price is a decrease in the medium-grained parallelization efficiency(see Fig. 5), and hence an increase in the local loss (see Fig. 2). A larger slot size will also improve the checkpointing as only information for the completed fragment calculations are saved (see Section 4 ).

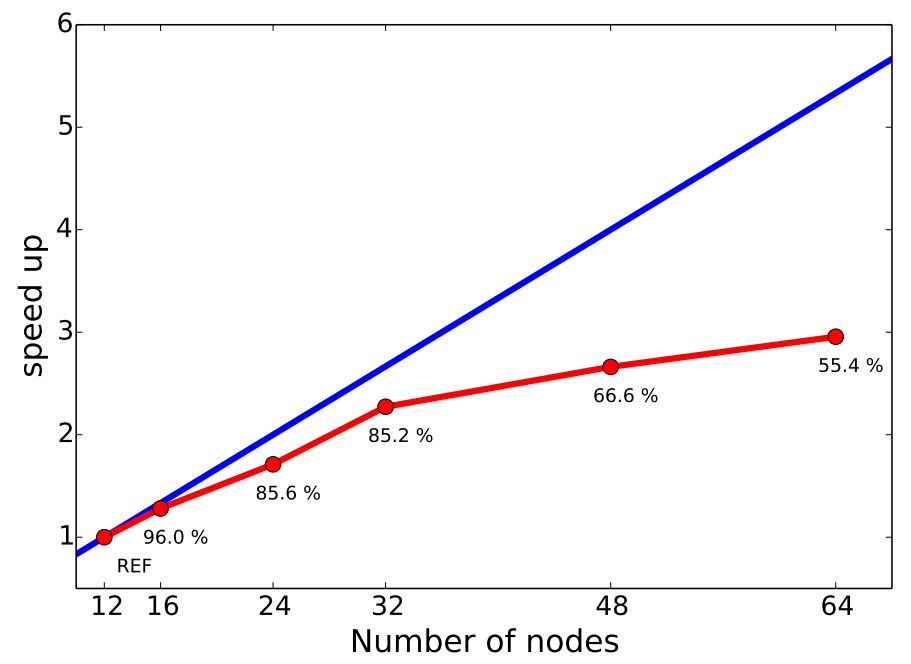

Figure 5. Strong scaling plot for the medium-grained parallelization: The largest pair fragment in $A A T_{10}$ using 12 to 64 Titan nodes. The numbers are efficiencies.

Fig. 6 shows the time-to-solution (TTS) for the different systems $\left\{A A T_{10}, A A T_{20}, A A T_{30}, A A T_{40}\right\}$ of increasing size using 14952 Titan nodes. For these systems, the size of the individual fragments is constant with respect to the total system size due to the local nature of electron correlation, and the computational cost is therefore mainly determined by the number of fragments. The proper treatment of dispersion effects (Section 2) means that the linear-scaling regime is approached asymptotically. In particular, the number of pair fragments considered for $A A T_{20}$ (33992) is slightly larger than twice the number of pair fragments in $A A T_{10}$ (16023), while the number of pair fragments considered for $A A T_{40}(69390)$ is $\frac{4}{3}$ times 


列

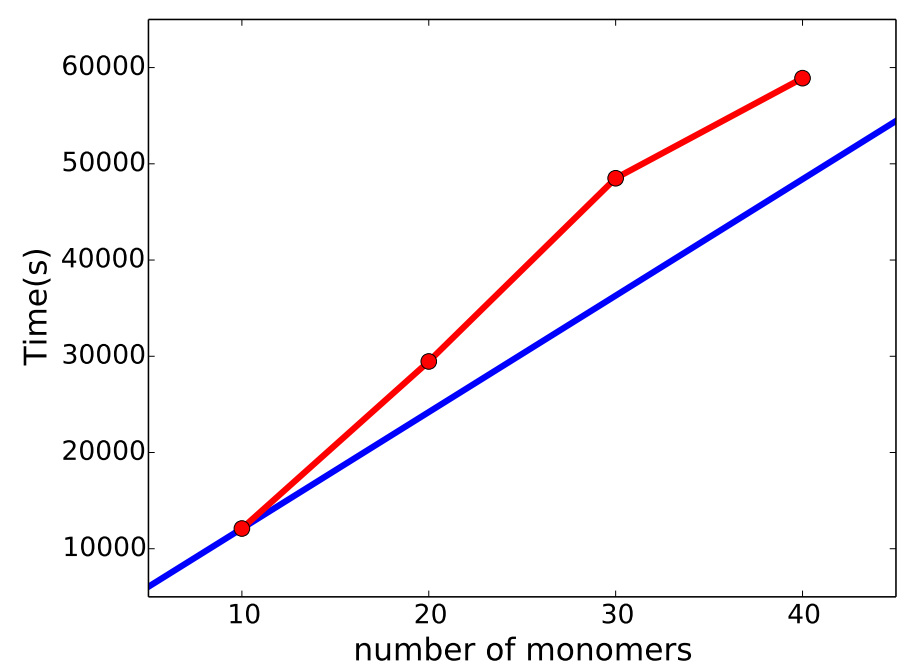

Figure 6. Computational scaling with system size for AATs of increasing lengths using 14952 Titan nodes. The blue line displays the ideal linear scaling behavior

In Table 4, we show weak scalability results for the different systems $\left\{A A T_{10}, A A T_{20}, A A T_{30}, A A T_{40}\right\}$ of increasing size, defining the weak scalability efficiency as $\mathrm{E}_{\mathrm{ws}}=F_{j} P_{i} T_{i} /\left(F_{i} P_{j} T_{j}\right)$ where $F_{i}, P_{i}$, and $T_{i}$ denote the FLOPs, number of processors and TTS for system $i$, respectively. We achieve a $83.1 \%$ weak scaling efficiency for $A A T_{40}$ relative to $A A T_{10}$ despite having to restart the $A A T_{40}$ calculation 6 times.

Table 4. Weak scalability results on Titan for AATs of different size using 3738 to 14952 nodes. The weak scaling efficiency $\mathbf{E}_{\mathrm{ws}}$ is given relative to $A A T_{10}$ and the last column denotes the number of times a calculation was restarted. The EFLOPs column denotes the accumulated number of EFLOPs performed during the entire simulation.

\begin{tabular}{cccccc}
\hline \hline System & \# nodes & EFLOPs & TTS(s) & $\mathrm{E}_{\mathrm{ws}}(\%)$ & restarted \\
\hline$A A T_{10}$ & 3738 & 4.08 & 47570 & & 1 \\
$A A T_{20}$ & 7476 & 9.41 & 60430 & 90.8 & 1 \\
$A A T_{30}$ & 11214 & 15.0 & 71180 & 81.9 & 10 \\
$A A T_{40}$ & 14952 & 19.4 & 68060 & 83.1 & 6 \\
\hline \hline
\end{tabular}

Each fragment calculation requires access to the Fock matrix and the $\mathrm{MO}$ coefficients. For $A A T_{40}$ this corresponds to 9.6 GB of data. The current scheme stores the data locally on the node which means that each MPI rank has less memory available for the $A A T_{40}$ calculation than for $A A T_{10}$ where this data only takes up 0.6 GB. This reduction in available memory results in more tiling and less efficient code which, together with 6 hardware failures, explains the non-ideal weak scalability. A better strategy is to store the required data in parallel distributed memory and retrieve it using one-sided MPI communication. This scheme will be operational shortly.

The weak scaling attribute arises from the linear scaling features of the DEC algorithm and ensures that we may treat molecular systems significantly larger than the conventional implementation. With its 24440 basis functions and 91280 auxiliary functions the $A A T_{40}$ RI-MP2 energy calculation is 2.5 times larger than the calculation of Katouda and Nakajima [16] using the K computer. The $A A T_{40}$ calculation was finished within 19 hours on Titan, using 14952 out of 18688 Titan nodes. In principle, DEC could easily be applied to larger molecular systems using larger basis sets, but the prerequisite HF calculation and orbital localization are currently limiting the size of the systems that may be treated. We are working on addressing these limitations.

inear-scaling behaviour in Fig. 6 can also be explained from the fact that the four calculations were restarted 1, 3, 6 , and 6 times, respectively.

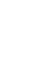




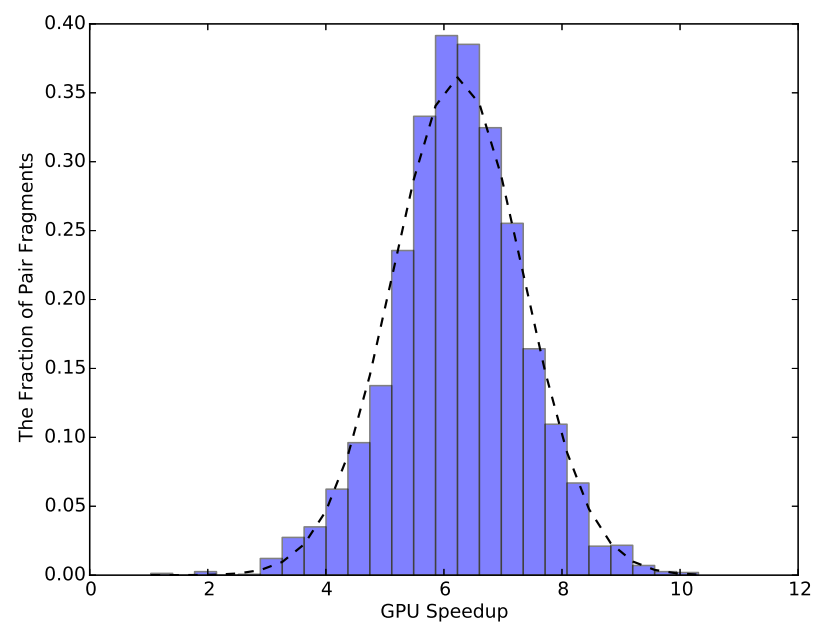

Figure 7. A histogram of GPU speedup for the individual pair fragments in $A A T_{4}$. The dotted line is the normal distribution using a standard deviation of 1.1 and a mean of 6.2

In a conventional calculation the fitting coefficients $C_{a i}^{\alpha}$ are written to disk and read in as needed. However, for $A A T_{40}$ this would require $49 \mathrm{~TB}$ of data (3400 valence orbitals, 19680 unoccupied orbitals and 91280 auxiliary functions) and put substantial strain on the file system. Alternatively, the fitting coefficients may be stored in parallel distributed memory, which again reduces the size of the operating memory. The DEC scheme eliminates this I/O and memory bottleneck. In a DEC calculation each fragment constructs the part of the fitting coefficients it needs, and stores in the reported calculation a maximum of $28 \mathrm{~GB}$ distributed among the nodes in the slot. It has been a design decision to limit the use of I/O despite this being a prominently used technique in quantum chemistry. The I/O is replaced by recalculation and parallel distributed memory storage, which makes the code portable and removes possible bottleneck issues in terms of disk requirements.

The speedup we have obtained through our use of GPUs may be estimated by performing an additional calculation without using the GPUs. For this purpose we chose the $A A T_{4}$ system. Fig. 7 displays a histogram of the GPU speedups for the individual pair fragments in the $A A T_{4}$ calculation. The maximum GPU speedup achieved is 10.3 while the average is 6.5, and the minimum speedup is 1.03. The general picture is that the GPU speedup is larger for larger fragments. This effectively reduces the inhomogeneity of the timings for the fragment calculations. The smallest speedup of 1.03 is achieved for the smallest fragment which takes 6.9 seconds using a single node, while the time for the largest fragment is reduced from 14 hours and 51 minutes to 2 hours and 0 minutes using 16 nodes. Since the distribution of fragment size for the larger $A A T \mathrm{~s}$ is similar to the one for $A A T_{4}$, the GPU performance for the larger AATs is similar to the ones obtained in the $A A T_{4}$ calculation.

We conclude that, unlike the conventional formulation, the DEC scheme is asymptotically linear scaling. Furthermore, the DEC scheme eliminates the I/O and memory bottleneck of the conventional approach. The linear scaling algorithm and the three levels of parallelization in our DEC implementation ensure strong and weak scalability. The hybrid cross-platform parallel implementation is well suited for achieving performance portability on current and future supercomputers.

\section{Conclusions and perspectives}

In the presented work, we have applied our recently developed DEC strategy using the lowest member of the CC hierarchy, the MP2 model, and in combination with the RI approximation demonstrated that DEC-RI-MP2 calculations may be carried out in a massively parallel manner. The combination of being both linearly scaling and massively parallel allows our DECRI-MP2 method to be applied to very large molecular systems. Our DEC-RI-MP2 implementation exhibits weak and strong scalability that ensures performance portability of the DEC-RI-MP2 code to the next generations of super computers. The largest calculations were carried out on a system comprising 2440 atoms, 6800 correlated electrons, 24440 basis functions, and 91280 auxiliary functions and using 14952 out of 18688 Titan nodes. The presented calculation is 2.5 times larger than the largest conventional RI-MP2 calculation [16. As DEC-RI-MP2 offers a full control of accuracy, it may serve as a supplement to standard DFT calculations and may become the method of choice for calculations on large molecular systems both with respect to the interpretation of experimental results and for reliable predictions of new structures and phenomena in many areas of molecular sciences. Our DEC strategy may also be applied to molecular gradients [103, 104] and higher members of the CC hierarchy and we are currently working on carrying out CCSD and CCSD(T) [105] calculations on large molecular systems using the DEC strategy.

The capabilities of our DEC strategy have been demonstrated here by carrying out DEC-RI-MP2 calculations of interaction 
energies of AATs using a cc-pVDZ basis set. To bring the calculation of interaction energies to the next level of accuracy, an extended basis set and a higher member of the CC hierarchy, e.g. $\operatorname{CCSD}(\mathrm{T})$, have to be used. Furthermore, to remove the basis set error, the DEC-CC methods have to be extended to include explicit electron-correlation corrections. We are currently also working along these lines.

The possibility of carrying out calculations using the DEC-CC hierarchy may be the start of a new era for hybrid, ONIOM-like, approaches [106]. In these approaches, methods of different quality are applied to different parts of a very large system, depending on the chemical importance of a given part of the system. Taking as an example an enzyme in solution, at the current stage of development of the hybrid approaches, an active site of an enzyme is typically treated with DFT, the backbone part of the enzyme with molecular mechanics (MM) or DFT, and the solvent surrounding the enzyme with MM. The DEC-CC hierarchy will make it possible to treat the active site with DEC-CCSD(T) and the backbone part with DEC-RI-MP2, leaving only the solvent part to an HF or MM treatment. As the protein backbone has been suggested to make important contributions to enzymatic catalysis [107, the possibility of addressing both the active site and the backbone part with quantum-mechanical correlated wave function methods may shift the modeling and understanding of enzymatic reactions to a new level.

The availability of the DEC-CCSD $(\mathrm{T})$ method will also enable the possibility of obtaining results for large molecular systems against which both DFT functionals, MM force fields, and other local correlation methods could be benchmarked. The DFT functionals and MM force fields are often benchmarked for small molecular systems, for which conventional $\operatorname{CCSD}(\mathrm{T})$ results are available. However, conclusions about the accuracy of the DFT functionals and MM force fields drawn from these benchmark studies on small systems do not necessarily carry over to large molecular systems.

Summarizing, the DEC-RI-MP2 massively parallel calculations reported here have been carried out on the Titan supercomputer at Oak Ridge Leadership Computing Facility utilizing both CPUs and GPUs and have shown strong and weak scalability. We have thus shown that the DEC strategy may effectively be used to remove the scaling wall for calculating the RI-MP2 correlation energy. We have also recently performed DEC calculations on other supercomputers and testing facilities in Europe and USA, including Piz Daint, Curie, and Vesta. The DEC program has been compiled and run successfully on all the aforementioned supercomputer architectures proving DEC to be cross-platform and easily portable.

\section{Acknowledgments}

This research used resources of the Oak Ridge Leadership Computing Facility at Oak Ridge National Laboratory, which is supported by the Office of Science of the Department of Energy under Contract DE-AC05-00OR22725. The DEC code has been ported to and developed on Titan as part of an Innovative and Novel Computational Impact on Theory and Experiment (INCITE) grant [108, 109, 110, and a through a Center for Accelerated Application Readiness (CAAR) [11] program. The research leading to these results has received funding from the European Research Council under the European Unions Seventh Framework Programme (FP/2007-2013)/ERC Grant Agreement no. 291371. DB acknowledges the Marie Curie Individual Fellowship funding for "DECOS", project number 657514. We thank Bobby Sumpter and Jacek Jakowski for useful discussions regarding the AATs.

[1] C. Møller, M. S. Plesset, Note on an approximation treatment for many-electron systems, Phys. Rev. 46 (7) (1934) 618.

[2] G. Purvis, R. J. Bartlett, a full coupled-cluster singles and doubles model - the inclusion of disconnected triples, J. Chem. Phys. 76 (1982) 1910.

[3] K. Raghavachari, G. W. Trucks, J. A. Pople, M. Head-Gordon, A fifth-order perturbation comparison of electron correlation theories, Chem. Phys. Lett. 157 (1989) 479.

[4] T. Helgaker, P. Jørgensen, J. Olsen, Molecular Electronic Structure Theory, First Edition, Wiley, Chichester, England, 2000.

[5] M. Ziółkowski, B. Jansík, T. Kjærgaard, P. Jørgensen, Linear scaling coupled cluster method with correlation energy based error control, J. Chem. Phys. 133 (2010) 014107. doi:10.1063/1.3456535.

[6] K. Kristensen, M. Ziółkowski, B. Jansík, T. Kjærgaard, P. Jørgensen, A locality analysis of the divideexpandconsolidate coupled cluster amplitude equations, J. Chem. Theory Comput. 7 (2011) 1677. doi:10.1021/ct200114k.

[7] P. Ettenhuber, P. Baudin, T. Kjærgaard, P. Jørgensen, K. Kristensen, Orbital spaces in the divide-expand-consolidate coupled cluster method, The Journal of Chemical Physics 144 (16). doi:http://dx.doi.org/10.1063/1.4947019. URL http://scitation.aip.org/content/aip/journal/jcp/144/16/10.1063/1.4947019

[8] J. L. Whitten, Coulombic potential energy integrals and approximations, J. Chem. Phys. 58 (10) (1973) $4496-4501$. doi:10.1063/1.1679012. URL http://scitation.aip.org/content/aip/journal/jcp/58/10/10.1063/1.1679012 
[9] B. I. Dunlap, J. W. D. Connolly, J. R. Sabin, On some approximations in applications of x $\alpha$ theory, J. Chem. Phys. 71 (8) (1979) 3396-3402.

[10] B. I. Dunlap, J. W. D. Connolly, J. R. Sabin, On first-row diatomic molecules and local density models, J. Chem. Phys. 71 (1979) 4993-4999.

[11] C. Van Alsenoy, Ab initio calculations on large molecules: The multiplicative integral approximation, Journal of Computational Chemistry 9 (6) (1988) 620-626. doi:10.1002/jcc.540090607

URL http://dx.doi.org/10.1002/jcc.540090607

[12] O. Vahtras, J. Almlöf, M. Feyereisen, Integral approximations for lcao-scf calculations, Chem. Phys. Lett. 213 (1993) 514.

[13] M. Feyereisen, G. Fitzgerald, A. Komornicki, Use of approximate integrals in ab initio theory. An application in MP2 energy calculations, Chem. Phys. Lett. 208 (1993) 359.

[14] D. E. Bernholdt, R. J. Harrison, Large-scale correlated electronic structure calculations: the ri-mp2 method on parallel computers, Chemical Physics Letters 250 (56) (1996) 477 - 484. doi:http://dx.doi.org/10.1016/0009-2614(96) 00054-1. URL http://www.sciencedirect.com/science/article/pii/0009261496000541

[15] M. Katouda, S. Nagase, Efficient parallel algorithm of second-order møller-plesset perturbation theory with resolutionof-identity approximation (ri-mp2), Int. J. Quantum Chem. 109 (2009) 2121. doi:10.1002/qua.22068

[16] M. Katouda, T. Nakajima, Mpi/openmp hybrid parallel algorithm of resolution of identity second-order møllerplesset perturbation calculation for massively parallel multicore supercomputers, J. Chem. Theory Comput. 9 (12) (2013) 5373-5380. doi:10.1021/ct400795v

[17] L. Vogt, R. Olivares-Amaya, S. Kermes, Y. Shao, C. Amador-Bedolla, A. Aspuru-Guzik, Accelerating resolution-ofthe-identity second-order mller-plesset quantum chemistry calculations with graphical processing units, Journal of Physical Chemistry A 112 (10) (2008) 2049-2057, cited By 67. doi:10.1021/jp0776762. URL http://www.scopus. com/inward/record.url?eid=2-s2.0-47049115273\&partnerID=40\&md5= 2b2a2f1b42fc00a19c8e4225c65ad394

[18] S. A. Maurer, J. Kussmann, C. Ochsenfeld, Communication: A reduced scaling j-engine based reformulation of sos-mp2 using graphics processing units, The Journal of Chemical Physics 141 (5) (2014) -. doi:http://dx.doi.org/10.1063/ 1.4891797 . URL http://scitation.aip.org/content/aip/journal/jcp/141/5/10.1063/1.4891797

[19] A. F. Izmaylov, G. E. Scuseria, Resolution of the identity atomic orbital laplace transformed second order moller-plesset theory for nonconducting periodic systems, Phys. Chem. Chem. Phys. 10 (2008) 3421-3429. doi:10.1039/B803274M. URL http://dx.doi.org/10.1039/B803274M

[20] T. Nakajima, K. Hirao, An approximate second-order mllerplesset perturbation approach for large molecular calculations, Chemical Physics Letters 427 (13) (2006) 225-229. doi:http://dx.doi.org/10.1016/j.cplett.2006.06.059 URL http://www.sciencedirect.com/science/article/pii/S0009261406009122

[21] R. Kendall, H. Frchtl, The impact of the resolution of the identity approximate integral method on modern ab initio algorithm development, Theoretical Chemistry Accounts 97 (1-4) (1997) 158-163, cited By 142. URL http://www. scopus. com/inward/record. url?eid=2-s2.0-0031285828\&partnerID=40\&md5= 3f6bb10774ab8f909f3c588a2cfc3bc7

[22] D. Cremer, Møller-Plesset perturbation theory: from small molecule methods to methods for thousands of atoms WIREs Comput. Mol. Sci. 1 (4) (2011) 509-530. doi:10.1002/wcms.58. URL http://onlinelibrary.wiley.com/doi/10.1002/wcms.58/abstract

[23] H. Koch, A. Sánchez de Merás, T. B. Pedersen, Reduced scaling in electronic structure calculations using cholesky decompositions, J. Chem. Phys. 118 (21) (2003) 9481-9484. doi:10.1063/1.1578621.

[24] F. Aquilante, R. Lindh, T. B. Pedersen, Unbiased auxiliary basis sets for accurate two-electron integral approximations, J. Chem. Phys. 127 (11) (2007) 114107. doi:10.1063/1.2777146. URL http://scitation.aip.org/content/aip/journal/jcp/127/11/10.1063/1.2777146

[25] F. Aquilante, L. Gagliardi, T. B. Pedersen, R. Lindh, Atomic cholesky decompositions: A route to unbiased auxiliary basis sets for density fitting approximation with tunable accuracy and efficiency, The Journal of Chemical Physics 130 (15) (2009) -. doi:http://dx.doi.org/10.1063/1.3116784. URL http://scitation.aip.org/content/aip/journal/jcp/130/15/10.1063/1.3116784 
[26] P. Pulay, Localizability of dynamic electron correlation, Chem. Phys. Lett. 100 (1983) 151.

[27] S. Saebø, P. Pulay, Local treatment of electron correlation, Ann. Rev. Phys. Chem. 44 (1) (1993) 213-236. doi: 10.1146/annurev.pc.44.100193.001241.

[28] P. Y. Ayala, G. E. Scuseria, Linear scaling second-order Møller-Plesset theory in the atomic orbital basis for large molecular systems, J. Chem. Phys. 110 (1999) 3660.

[29] G. E. Scuseria, P. Y. Ayala, Linear scaling coupled cluster and perturbation theories in the atomic orbital basis, J. Chem. Phys. 111 (1999) 8330.

[30] D. S. Lambrecht, B. Doser, C. Ochsenfeld, Rigorous integral screening for electron correlation methods, J. Chem. Phys. 123 (18) (2005) 184102.

[31] C. Hampel, H.-J. Werner, Local treatment of electron correlation in coupled cluster theory, J. Chem. Phys. 104 (1996) 6286.

[32] M. Schütz, G. Hetzer, H.-J. Werner, Low-order scaling local electron correlation methods. I. Linear scaling local MP2, J. Chem. Phys. 111 (13) (1999) 5691-5705. doi:10.1063/1.479957.

[33] G. Hetzer, M. Schütz, H. Stoll, H.-J. Werner, Low-order scaling local correlation methods ii: Splitting the coulomb operator in linear scaling local second-order møller-plesset perturbation theory, J. Chem. Phys. 113 (21) (2000) 94439455.

[34] J. E. Subotnik, A. Sodt, M. Head-Gordon, A near linear-scaling smooth local coupled cluster algorithm for electronic structure, J. Chem. Phys. 125 (7) (2006) 074116.

[35] J. Friedrich, M. Dolg, Fully Automated Incremental Evaluation of MP2 and CCSD(T) Energies: Application to Water Clusters, J. Chem. Theory Comput. 5 (2) (2009) 287-294. doi:10.1021/ct800355e URL http://dx.doi.org/10.1021/ct800355e

[36] F. Neese, F. Wennmohs, A. Hansen, Efficient and accurate local approximations to coupled-electron pair approaches: An attempt to revive the pair natural orbital method, J. Chem. Phys. 130 (2009) 114108.

[37] F. Neese, A. Hansen, D. G. Liakos, Efficient and accurate approximations to the local coupled cluster singles doubles method using a truncated pair natural orbital basis, J. Chem. Phys. 131 (2009) 064103.

[38] W. Li, P. Piecuch, Improved design of orbital domains within the cluster-in-molecule local correlation framework: Single-environment cluster-in-molecule ansatz and its application to local coupled-cluster approach with singles and doubles, J. Phys. Chem. A 114 (2010) 8644.

[39] H.-J. Werner, M. Schütz, An efficient local coupled cluster method for accurate thermochemistry of large systems, J. Chem. Phys. 135 (2011) 144116.

[40] Y. Kurashige, J. Yang, G. K.-L. Chan, F. R. Manby, Optimization of orbital-specific virtuals in local møller-plesset perturbation theory, J. Chem. Phys. 136 (12) (2012) 124106.

[41] C. Riplinger, F. Neese, An efficient and near linear scaling pair natural orbital based local coupled cluster method, J. Chem. Phys. 138 (2013) 034106.

[42] Y. Guo, W. Li, D. Yuan, S. Li, The relative energies of polypeptide conformers predicted by linear scaling second-order mller-plesset perturbation theory, Science China Chemistry 57 (10) (2014) 1393-1398.

[43] Y. Guo, W. Li, S. Li, Improved Cluster-in-Molecule Local Correlation Approach for Electron Correlation Calculation of Large Systems, J. Phys. Chem. A 118 (39) (2014) 8996-9004. doi:10.1021/jp501976x. URL http: //dx.doi.org/10.1021/jp501976x

[44] P. Pinski, C. Riplinger, E. F. Valeev, F. Neese, Sparse maps-a systematic infrastructure for reduced-scaling electronic structure methods. i. an efficient and simple linear scaling local mp2 method that uses an intermediate basis of pair natural orbitals, J. Chem. Phys. 143 (3) (2015) 034108.

[45] K. Kristensen, T. Kjærgaard, I.-M. Høyvik, P. Ettenhuber, P. Jørgensen, B. Jansík, S. Reine, J. Jakowski, The divideexpandconsolidate mp2 scheme goes massively parallel, Mol. Phys. 111 (2013) 1196. doi:10.1080/00268976. 2013.783941.

[46] P. Baudin, P. Ettenhuber, S. Reine, K. Kristensen, T. Kjærgaard, Efficient linear-scaling second-order møller-plesset perturbation theory: The divide-expand-consolidate ri-mp2 model, J. Chem. Phys. 144 (5) (2016) 054102. doi: $10.1063 / 1.4940732$. 
[47] H.-J. Werner, F. R. Manby, P. J. Knowles, Fast linear scaling second-order møller-plesset perturbation theory (mp2) using local and density fitting approximations, The Journal of chemical physics 118 (18) (2003) 8149-8160.

[48] H.-J. Werner, G. Knizia, C. Krause, M. Schwilk, M. Dornbach, Scalable electron correlation methods i.: Pno-lmp2 with linear scaling in the molecular size and near-inverse-linear scaling in the number of processors, Journal of Chemical Theory and Computation 11 (2) (2015) 484-507.

[49] S. Li, J. Shen, W. Li, Y. Jiang, An efficient implementation of the cluster-in-molecule approach for local electron correlation calculations, The Journal of chemical physics 125 (7) (2006) 074109.

[50] H. Stoll, The correlation energy of crystalline silicon, Chem. Phys. Lett. 191 (1992) 548.

[51] M. Kobayashi, Y. Imamura, H. Nakai, Alternative linear-scaling methodology for the second-order Mller-Plesset perturbation calculation based on the divide-and-conquer method, J. Chem. Phys. 127 (7) (2007) 074103. doi: $10.1063 / 1.2761878$.

[52] M. Katouda, M. Kobayashi, H. Nakai, S. Nagase, Two-level hierarchical parallelization of second-order møllerplesset perturbation calculations in divide-and-conquer method, J. Comput. Chem. 32 (2011) 2756.

[53] T. Ishikawa, K. Kuwata, Fragment molecular orbital calculation using the ri-mp2 method, Chem. Phys. Lett. 474 (2009) 195.

[54] Y. Mochizuki, K. Yamashita, T. Murase, T. Nakano, K. Fukuzawa, K. Takematsu, H. Watanabe, S. Tanaka, Large scale FMO-MP2 calculations on a massively parallel-vector computer, Chem. Phys. Lett. 457 (46) (2008) 396-403. doi:10.1016/j.cplett.2008.03.090

[55] V. Deev, M. A. Collins, Approximate ab initio energies by systematic molecular fragmentation., J. Chem. Phys. 122 (15) (2005) 154102.

[56] X. He, J. Z. H. Zhang, The generalized molecular fractionation with conjugate caps/molecular mechanics method for direct calculation of protein energy, The Journal of Chemical Physics 124 (18). doi:http://dx.doi.org/10.1063/1. 2194535. URL http://scitation.aip.org/content/aip/journal/jcp/124/18/10.1063/1.2194535

[57] W. Li, S. Li, Y. Jiang, Generalized energy-based fragmentation approach for computing the ground-state energies and properties of large molecules, The Journal of Physical Chemistry A 111 (11) (2007) 2193-2199, pMID: 17388268. arXiv:http://dx.doi.org/10.1021/jp067721q, doi:10.1021/jp067721q URL http://dx.doi.org/10.1021/jp067721q

[58] A. P. Rahalkar, M. Katouda, S. R. Gadre, S. Nagase, Molecular tailoring approach in conjunction with MP2 and Ri-MP2 codes: A comparison with fragment molecular orbital method, J. Comput. Chem. 31 (13) (2010) 2405-2418.

[59] H.-A. Le, H.-J. Tan, J. F. Ouyang, R. P. A. Bettens, Combined fragmentation method: A simple method for fragmentation of large molecules, Journal of Chemical Theory and Computation 8 (2) (2012) 469-478, pMID: 26596597. arXiv:http://dx.doi.org/10.1021/ct200783n, doi:10.1021/ct200783n. URL http://dx.doi.org/10.1021/ct200783n

[60] S. Li, W. Li, J. Ma, Generalized energy-based fragmentation approach and its applications to macromolecules and molecular aggregates, Accounts of Chemical Research 47 (9) (2014) 2712-2720, pMID: 24873495. arXiv: http://dx. doi.org/10.1021/ar500038z, doi:10.1021/ar500038z. URL http://dx.doi.org/10.1021/ar500038z

[61] J. Almlöf, Elimination of energy denominators in Møller-Plesset perturbation theory by a laplace transform approach, Chem. Phys. Lett. 181 (1991) 319.

[62] M. Häser, Møller-Plesset perturbation theory for large molecules, Theor Chim Acta 87 (1993) 147.

[63] B. Doser, D. S. Lambrecht, J. Kussmann, C. Ochsenfeld, Linear-scaling atomic orbital-based second-order MllerPlesset perturbation theory by rigorous integral screening criteria, J. Chem. Phys. 130 (6) (2009) 064107. doi:10.1063/1. 3072903

[64] S. A. Maurer, D. S. Lambrecht, J. Kussmann, C. Ochsenfeld, Efficient distance-including integral screening in linearscaling Møller-Plesset perturbation theory, J. Chem. Phys. 138 (1) (2013) 014101. doi:10.1063/1.4770502. URL http://scitation.aip.org/content/aip/journal/jcp/138/1/10.1063/1.4770502 
[65] B. Doser, J. Zienau, L. Clin, D. S. Lambrecht, C. Ochsenfeld, A linear-scaling mp2 method for large molecules by rigorous integral screening criteria, Zeitschrift fr Physikalische Chemie International journal of research in physical chemistry and chemical physics 224 (3) (2010) 397.

[66] M. Ziółkowski, B. Jansík, P. Jørgensen, J. Olsen, Maximum locality in occupied and virtual spaces using a least-change strategy, J. Chem. Phys. 131 (2009) 124112.

[67] B. Jansík, S. Høst, K. Kristensen, P. Jørgensen, Local orbitals by minimizing powers of the orbital variance, J. Chem. Phys. 134 (19) (2011) 194104.

[68] I.-M. Høyvik, B. Jansík, P. Jørgensen, Orbital localization using fourth central moment minimization, J. Chem. Phys. 137 (22) (2012) 224114.

[69] I.-M. Høyvik, B. Jansík, P. Jørgensen, Trust region minimization of orbital localization functions, J. Chem. Theory Comput. 8 (2012) 3137

[70] I.-M. Høyvik, B. Jansík, P. Jørgensen, Pipekmezey localization of occupied and virtual orbitals, J. Comput. Chem. 34 (17) (2013) 1456-1462.

[71] I.-M. Høyvik, K. Kristensen, T. Kjærgaard, P. Jørgensen, A perspective on the localizability of hartree-fock orbitals, Theor. Chem. Acc. 133 (1) (2013) 1417.

[72] S. F. Boys, Construction of some molecular orbitals to be approximately invariant for changes from one molecule to another, Rev. Mod. Phys. 32 (2) (1960) 296-299.

[73] J. M. Foster, S. F. Boys, Canonical configurational interaction procedure, Rev. Mod. Phys. 32 (1960) 300.

[74] C. Edmiston, K. Ruedenberg, Localized atomic and molecular orbitals, Rev. Mod. Phys. 35 (1963) 457.

[75] C. Edmiston, K. Ruedenberg, Localized atomic and molecular orbitals. ii, J. Chem. Phys. 43 (10) (1965) S97-S116.

[76] V. Magnasco, A. Perico, Uniform localization of atomic and molecular orbitals. i, J. Chem. Phys. 47 (3) (1967) $971-981$.

[77] J. Pipek, P. G. Mezey, A fast intrinsic localization procedure applicable for ab initio and semiempirical linear combination of atomic orbital wave functions, J. Chem. Phys. 90 (1989) 4916.

[78] J. Pipek, Localization measure and maximum delocalization in molecular systems, Int. J. Quantum Chem. 36 (1989) 487.

[79] D. Maynau, S. Evangelisti, N. Guihéry, C. J. Calzado, J.-P. Malrieu, Direct generation of local orbitals for multireference treatment and subsequent uses for the calculation of the correlation energy, J. Chem. Phys. 116 (2002) 10060.

[80] J. E. Subotnik, A. D. Dutoi, M. Head-Gordon, Fast localized orthonormal virtual orbitals which depend smoothly on nuclear coordinates, J. Chem. Phys. 123 (2005) 114108.

[81] F. Aquilante, T. B. Pedersen, A. S. de Merás, H. Koch, Fast noniterative orbital localization for large molecules, J. Chem. Phys. 125 (17) (2006) 174101.

[82] C. Zhang, S. Li, An efficient localization procedure for large systems using a sequential transformation strategy, J. Chem. Phys. 141 (24) (2014) 244106.

[83] I.-M. Hyvik, P. Jrgensen, Characterization and generation of local occupied and virtual hartreefock orbitals, Chemical Reviews 116 (5) (2016) 3306-3327, pMID: 26855066. arXiv:http://dx.doi.org/10.1021/acs.chemrev.5b00492, doi:10.1021/acs.chemrev.5b00492 URL http://dx.doi.org/10.1021/acs.chemrev.5b00492

[84] E. Schwegler, M. Challacombe, Linear scaling computation of the hartree-fock exchange matrix, J. Chem. Phys. 105 (7) (1996) 2726.

[85] E. Schwegler, M. Challacombe, M. Head-Gordon, Linear scaling computation of the fock matrix. ii. rigorous bounds on exchange integrals and incremental fock build, J. Chem. Phys. 106 (23) (1997) 9708.

[86] M. Challacombe, E. Schwegler, Linear scaling computation of the fock matrix, J. Chem. Phys. 106 (13) (1997) 5526.

[87] C. Ochsenfeld, C. A. White, M. Head-Gordon, Linear and sublinear scaling formation of hartree-fock-type exchange matrices, J. Chem. Phys. 109 (5) (1998) 1663.

[88] C. A. White, B. G. Johnson, P. M. W. Gill, M. Head-Gordon, The continuous fast multipole method, Chem. Phys. Lett. 230 (1-2) (1994) 8. 
[89] M. C. Strain, G. E. Scuseria, M. J. Frisch, Achieving linear scaling for the electronic quantum coulomb problem, Science 271 (1996) 51.

[90] P. Sałek, S. Høst, L. Thøgersen, P. Jørgensen, P. Manninen, J. Olsen, B. Jansík, S. Reine, F. Pawłowski, E. Tellgren, T. Helgaker, S. Coriani, Linear-scaling implementation of molecular electronic self-consistent field theory, J. Chem. Phys. 126 (11) (2007) 114110.

[91] S. Goedecker, Linear scaling electronic structure methods, Rev. Mod. Phys. 71 (4) (1999) 1085. doi:10.1103/ RevModPhys.71.1085.

[92] S. Goedecker, G. E. Scuseria, Linear scaling electronic structure methods in chemistry and physics, Comp. Sci. Eng. 5 (4) (2003) 14.

[93] A. P. Rendell, Diagonalization-free scf, Chemical Physics Letters 229 (3) (1994) 204 - 210. doi:http://dx.doi.org/ 10.1016/0009-2614(94)01053-6. URL http://www.sciencedirect.com/science/article/pii/0009261494010536

[94] E. Rudberg, E. H. Rubensson, P. Saek, Hartreefock calculations with linearly scaling memory usage, The Journal of Chemical Physics 128 (18). doi:http://dx.doi.org/10.1063/1.2918357. URL http://scitation.aip.org/content/aip/journal/jcp/128/18/10.1063/1.2918357

[95] I.-M. Høyvik, K. Kristensen, B. Jansík, P. Jørgensen, The divide-expand-consolidate family of coupled cluster methods: Numerical illustrations using second order mller-plesset perturbation theory, J. Chem. Phys. 136 (2012) 014105. doi: 10.1063/1.3667266.

[96] B. Sumpter, V. Meunier, A. Vazquez-Mayagoitia, R. Castellano, Investigation of the nanoscale self-assembly of donorsigma-acceptor molecules, Int. J. Quantum Chem. 107 (2007) 2233. doi:10.1002/qua.21411.

[97] B. Sumpter, V. Meunier, E. Valeev, A. J. Lampkins, H. Li, R. K. Castellano, A new class of supramolecular wires, J. Phys. Chem. C 111 (2007) 18912. doi:10.1021/jp076329p.

[98] F. Weigend, A. Köhn C. Hättig, Efficient use of the correlation consistent basis sets in resolution of the identity MP2 calculations, J. Chem. Phys. 116 (2002) 3175. doi:10.1063/1.1445115

[99] S. F. Boys, F. Bernardi, The calculation of small molecular interactions by the differences of separate total energies. some procedures with reduced errors, Mol. Phys. 19 (1970) 553. doi:10.1080/00268977000101561.

[100] J. Dongarra, K. London, S. Moore, P. Mucci, D. Terpstra, Using PAPI for Hardware Performance Monitoring on Linux Systems. In Conference on Linux Clusters: The HPC Revolution, Linux Clusters Institute, Urbana, Illinois, 2002.

[101] LSDALTON, a linear-scaling molecular electronic structure program, Release Dalton2016, http://daltonprogram.org (2016).

[102] K. Aidas, C. Angeli, K. L. Bak, V. Bakken, R. Bast, L. Boman, O. Christiansen, R. Cimiraglia, S. Coriani, P. Dahle, E. K. Dalskov, U. Ekström, T. Enevoldsen, J. J. Eriksen, P. Ettenhuber, B. Fernández, L. Ferrighi, H. Fliegl, L. Frediani, K. Hald, A. Halkier, C. Hättig, H. Heiberg, T. Helgaker, A. C. Hennum, H. Hettema, E. Hjertenæs, S. Høst, I.-M. Høyvik, M. F. Iozzi, B. Jansik, H. J. A. Jensen, D. Jonsson, P. Jørgensen, J. Kauczor, S. Kirpekar, T. Kjærgaard, W. Klopper, S. Knecht, R. Kobayashi, H. Koch, J. Kongsted, A. Krapp, K. Kristensen, A. Ligabue, O. B. Lutnæs, J. I. Melo, K. V. Mikkelsen, R. H. Myhre, C. Neiss, C. B. Nielsen, P. Norman, J. Olsen, J. M. H. Olsen, A. Osted, M. J. Packer, F. Pawlowski, T. B. Pedersen, P. F. Provasi, S. Reine, Z. Rinkevicius, T. A. Ruden, K. Ruud, V. Rybkin, P. Salek, C. C. M. Samson, A. S. de Merás, T. Saue, S. P. A. Sauer, B. Schimmelpfennig, K. Sneskov, A. H. Steindal, K. O. Sylvester-Hvid, P. R. Taylor, A. M. Teale, E. I. Tellgren, D. P. Tew, A. J. Thorvaldsen, L. Thøgersen, O. Vahtras, M. A. Watson, D. J. D. Wilson, M. Ziolkowski, H. Ågren, The dalton quantum chemistry program system, WIREs Comput. Mol. Sci. 4 (2013) 269. doi:10.1002/wcms.1172.

[103] K. Kristensen, P. Jørgensen, B. Jansík, T. Kjærgaard, S. Reine, Molecular gradient for second-order møller-plesset perturbation theory using the divide-expand-consolidate (dec) scheme, J. Chem. Phys. 137 (2012) 114102.

[104] D. Bykov, K. Kristensen, T. Kjærgaard, The molecular gradient using the divide-expand-consolidate resolution of the identity second-order møller-plesset perturbation theory: The dec-ri-mp2 gradient, J. Chem. Phys. 145 (2). doi : http: //dx.doi.org/10.1063/1.4956454. URL http://scitation.aip.org/content/aip/journal/jcp/145/2/10.1063/1.4956454

[105] J. J. Eriksen, P. Baudin, P. Ettenhuber, K. Kristensen, T. Kjærgaard, P. Jørgensen, Linear-scaling coupled cluster with perturbative triple excitations: The divideexpandconsolidate ccsd(t) model, J. Chem. Theory Comput. 11 (7) (2015) 2984-2993. doi:10.1021/acs . jctc.5b00086 
[106] T. Vreven, K. S. Byun, I. Komáromi, S. Dapprich, J. A. Montgomery, K. Morokuma, M. J. Frisch, Combining Quantum Mechanics Methods with Molecular Mechanics Methods in ONIOM, J. Chem. Theory Comput. 2 (3) (2006) 815-826. doi:10.1021/ct050289g.

[107] G. A. Cisneros, M. Wang, P. Silinski, M. C. Fitzgerald, W. Yang, The Protein Backbone Makes Important Contributions to 4-Oxalocrotonate Tautomerase Enzyme Catalysis: Understanding from Theory and Experiment, Biochemistry 43 (22) (2004) 6885-6892. doi:10.1021/bi049943p.

[108] The innovative and novel computational impact on theory and experiment (INCITE) program 2014, https://www . olcf .ornl.gov/leadership-science/2014-incite-projects/.

109] The innovative and novel computational impact on theory and experiment (INCITE) program 2015, https://www . olcf.ornl.gov/leadership-science/2015-incite-projects/.

[110] The innovative and novel computational impact on theory and experiment (INCITE) program 2016, https://www.

[111] Center for accelerated application readiness (CAAR), https://www.olcf.ornl.gov/caar. 